Published in final edited form as:

Neuroimage. 2018 November 01; 181: 120-131. doi:10.1016/j.neuroimage.2018.06.080.

\title{
Distinct neural processes for the perception of familiar versus unfamiliar faces along the visual hierarchy revealed by EEG
}

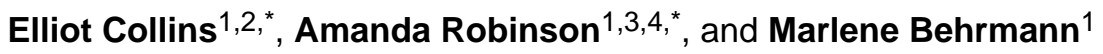 \\ ${ }^{1}$ Department of Psychology and Center for the Neural Basis of Cognition, Carnegie Mellon \\ University \\ ${ }^{2}$ School of Medicine, University of Pittsburgh, Pittsburgh, USA \\ ${ }^{3}$ School of Psychology, The University of Sydney \\ ${ }^{4}$ Department of Cognitive Science, Macquarie University
}

\section{Abstract}

Humans recognize faces with ease, despite the complexity of the task and of the visual system which underlies it. Different spatial regions, including both the core and extended face processing networks, and distinct temporal stages of processing have been implicated in face recognition, but there is ongoing controversy regarding the extent to which the mechanisms for recognizing a familiar face differ from those for an unfamiliar face. Here, we used electroencephalogram (EEG) and flicker SSVEP, a high signal-to-noise approach, and searchlight decoding methods to elucidate the mechanisms mediating the processing of familiar and unfamiliar faces in the time domain. Familiar and unfamiliar faces were presented periodically at $15 \mathrm{~Hz}, 6 \mathrm{~Hz}$ and $3.75 \mathrm{~Hz}$ either upright or inverted in separate blocks, with the rationale that faster frequencies require shorter processing times per image and tap into fundamentally different levels of visual processing. The $15 \mathrm{~Hz}$ trials, likely to reflect early visual processing, exhibited enhanced neural responses for familiar over unfamiliar face trials, but only when the faces were upright. In contrast, decoding methods revealed similar classification accuracies for upright and inverted faces for both familiar and unfamiliar faces. For the $6 \mathrm{~Hz}$ frequency, familiar faces had lower amplitude responses than unfamiliar faces, and decoding familiarity was more accurate for upright compared with inverted faces. Finally, the $3.75 \mathrm{~Hz}$ frequency revealed no main effects of familiarity, but decoding showed significant correlations with behavioral ratings of face familiarity, suggesting that activity evoked by this slow presentation frequency reflected higher-level, cognitive aspects of familiarity processing. This three-way dissociation between frequencies reveals that fundamentally different stages of the visual hierarchy are modulated by face familiarity. The combination of experimental and analytical approaches used here represent a novel method for elucidating spatio-temporal characteristics within the visual system.

Address: Carnegie Mellon University, 5000 Forbes Ave, Pittsburgh, PA, 15213, USA, Phone: (412) 268-4237, Elliot Collins < eggcollin@andrew.cmu.edu>.

Shared contribution, order is alphabetical

Publisher's Disclaimer: This is a PDF file of an unedited manuscript that has been accepted for publication. As a service to our customers we are providing this early version of the manuscript. The manuscript will undergo copyediting, typesetting, and review of the resulting proof before it is published in its final form. Please note that during the production process errors may be discovered which could affect the content, and all legal disclaimers that apply to the journal pertain. 


\section{Keywords}

face processing; face recognition; FPVS; frequency tagging; EEG; famous faces

\section{Introduction}

Despite the necessity to discriminate among thousands of homogenous exemplars (Bruce, Henderson, Newman, \& Burton, 2001), human face recognition is remarkably rapid and accurate across highly variable contexts and under challenging viewing conditions. One striking index of human face expertise is the face inversion effect, the finding that recognition is severely impaired when faces are inverted (Farah, Tanaka, \& Drain, 1995; Freire, Lee, Symons, 2000; Megreya \& Burton, 2006). Familiarity of individual faces is another marker of face recognition; for upright faces, recognition is better (Burton, 2013) and faster (Ramon, Vizioli, Liu-Shuang, \& Rossion, 2015) for familiar than for unfamiliar faces. Furthermore, familiar faces are matched more rapidly than unfamiliar faces (Kramer, Manesi, Towler, Reynolds, \& Burton, 2017) and are robust to variations in viewpoint and image format (Freiwald, Duchaine, \& Yovel, 2016). There is ongoing debate concerning the representations that permit this advantage for familiar versus unfamiliar face recognition because the neural mechanisms that allow such flexible face recognition are highly complex. To dissociate familiar and unfamiliar face processing more broadly, a fine-grained understanding of the neural mechanisms of face recognition is paramount.

Face recognition is known to recruit a distributed finely-tuned neural network, which is perhaps unsurprising, given the complexity of the stimulus and contributions of perceptual and semantic information. Across the human brain, these regions include both core, visual perceptual regions, and extended, person knowledge regions (Haxby, Hoffman, \& Gobbini, 2000; Gobbini \& Haxby, 2007), but it is unclear to what extent each node in the network is involved in face processing generally rather than face recognition specifically. Collectively, the evidence suggests that the posterior/core regions in the face network such as the occipital face area (OFA) and fusiform face area (FFA) are involved in representing the more structural/visual aspects of face processing (for example (Davies-Thompson, Gouws, \& Andrews, 2009; Ewbank \& Andrews, 2008)), in contrast with the more anterior/extended regions of the face network that subserve person knowledge (anterior paracingulate, posterior STS/TPJ, anterior temporal cortex, precuneus), and emotion-related areas (amygdala, insula, striatum/reward system) (Gobbini \& Haxby, 2007; Guntupalli, Wheeler, $\&$ Gobbini, 2017). With respect to differential performance for familiar versus unfamiliar faces, detailed investigations have uncovered spatial and temporal differences particularly within the extended face network, but some effects have also been reported within the core face network, as well. As will be evident below, many open questions remain concerning the neural mechanisms of face recognition.

\section{Spatial differences in face recognition network}

Using functional MRI, studies have shown differences for familiar versus unfamiliar faces in many brain regions across both the core and extended face processing networks. In the core network, weaker BOLD activations for familiar compared to unfamiliar faces have been 
observed in right occipito-temporal regions (Dubois et al. 1999; Rossion et al. 2001; Henson et al. 2000), but other studies revealed the opposite pattern (Leveroni et al. 2000; Rotshtein et al. 2005). In contrast to these discrepant results, there is greater consistency in findings in the extended system, revealing a differential response to familiar versus unfamiliar faces (Gorno-Tempini et al. 1998; Leveroni et al. 2000; Nakamura et al. 2000; Rotshtein et al. 2005; Sergent et al. 1992; Landi \& Freiwald, 2017). In one study, when high spatial frequency information was gradually introduced to the input image, activation in core occipitotemporal regions increased with visual information but medial temporal lobe structures (perirhinal cortex, amygdala, hippocampus) and anterior inferior temporal cortex responded abruptly when sufficient information for familiar face recognition was accumulated, primarily implicating the extended network in face recognition (Ramon et al., 2015) (see also (Barense, Henson, \& Graham, 2011)). Relatedly, two newly-identified regions, the temporal pole and perirhinal cortex, are also activated in a non-linear fashion for familiar versus unfamiliar faces or objects, as revealed in a recent study with nonhuman primates (Landi \& Freiwald, 2017).

Many possible explanations may account for the differences in responses between core and extended systems. Viewing familiar versus unfamiliar faces is likely to involve differences in multiple facets of perception due to attention, pattern recognition, and other cognitive factors such as emotion and identity processing, all of which might also vary depending on the nature of the required task. Furthermore, many of these processes have proven difficult to separate using fMRI because the BOLD signal does not provide sufficient temporal resolution to assess the propagation of information along the ventral occipital temporal cortex in real time.

\section{Temporal differences in familiar versus unfamiliar face processing}

In light of the limitations of BOLD studies in uncovering the temporal dynamics of face perception, investigations utilizing scalp event-related potentials (ERPs) have attempted to clarify the dynamic relationship between early and late stages of processing in the face network (Sadeh, Podlipsky, Zhdanov, \& Yovel, 2010). These electrophysiological measures have revealed differential responses to faces over other objects and effects of variation in face viewpoint are evident early (sub-200ms), involving the core face network (e.g., Eimer, 2000). The later $\mathrm{N} 250$ and $\mathrm{P} 300$ components have generally been associated with higherlevel, view-invariant processes such as integration with semantic and name information (Tanaka et al., 2006) and emotion perception (Campanella et al., 2013), and are therefore characteristic of the extended face network. Elucidating the temporal signatures of face recognition is also critical because participants respond faster to familiar than unfamiliar faces (Ramon et al., 2015). Face inversion typically delays and increases the N170 component (Rossion et al 2000; Itier \& Taylor, 2002; Jacques et al 2007), implicating the core face network in one aspect of face recognition, but there is little evidence for familiarity effects during early visual processing. In contrast, face familiarity modulates the amplitude of the ERP waveform around N250ms (Henson, Mouchlianitis, Matthews, Kouider, 2008; Kaufmann, Schweinberger, \& Burton, 2009; Schweinberger, Pickering, Jentzsch, Burton, \& Kaufmann, 2002), and the magnitude of this component correlates with recognition speed for upright faces (Huang et al., 2017) as well as for learned faces (Kaufmann, 
Schweinberger, \& Burton, 2009; Kaufmann \& Schweinberger, 2012; Huang et al., 2017). The later P300 component is also influenced by face familiarity and it, too, is strongly correlated with behavior (Hanso, Bachmann, \& Murd, 2010).

As evident from the above, familiarity appears to impact stages of processing differentially, with more consistent modulation of later ERP components. It is possible, however, that ERP techniques used thus far are not sufficiently powerful to detect subtle changes in the processing within the core face network. Whole brain electrophysiological methods, as adopted in the current study, offer the potential to reconcile discrepancies in the previous literature on familiar versus unfamiliar face processing from both fMRI and ERP methods and to uncover the influence of familiarity at different stages of processing.

\section{Signatures of face processing revealed by frequency tagging}

One useful approach for exploring face-specific neural signatures utilizes periodic visual stimulation. Presenting visual stimuli periodically at a specific frequency results in neural responses with the same frequency, which can be measured with EEG, an effect traditionally called the steady-state visual evoked response (SSVEP; Regan 1966). This approach has recently been termed fast periodic visual stimulation (FPVS; Rossion 2014), typically for "oddball" paradigms, whereby a stimulus or category repeats at a slower frequency within a periodic stream (e.g. Rossion et al 2015). Neural responses to repetitive visual stimuli have the potential to tap into different neural processes with different scalp topographies depending on the stimulation frequency. Face-related responses are largest for the $5.88 \mathrm{~Hz}$ presentation rate, corresponding exactly to $170 \mathrm{~ms}$ presentation per face, with a similar topographic pattern over right temporal-occipital electrodes at the N170 ERP component (Alonso-Prieto et al., 2013). More generally, rapid serial presentation of stimuli has revealed a number of important findings with respect to signal amplitude, including hemisphericspecific differences in enhancement in response to attention (Quek, Nemdorov, Rossion, Liu-Shuang, 2018), FPVS response immunity to temporal predictability (Quek \& Rossion, 2017), and individual differences in FPVS responses as a marker of overall face recognition ability (Xu, Liu-Shuang, Rossion, \& Tanaka, 2017). Note, however, that electrophysiological dynamics are not always reflected in greater magnitude in the frequency domain. In the case of adaptation, where images of the same face are repeated, the evoked EEG amplitude is suppressed (Rossion \& Boremanse, 2011; Nemrodov, Jacques, \& Rossion, 2015; Retter \& Rossion, 2017). The presence of adaptation effects at $6 \mathrm{~Hz}$ stimulation frequencies implicates the core face network (e.g., within right FFA) in identity recognition.

FPVS effects at other frequencies have typically been used to study early visual processes and attention. Fast frequency presentations $(8-20 \mathrm{~Hz}$ or $50-125 \mathrm{~ms}$ per image) result in large neural responses and are associated with early visual cortex activity, likely because each successive image masks and limits the processing of the previous image (Di Russo et al 2007, Robinson et al 2017). Stimuli tagged with such frequencies typically evoke larger amplitudes when in the setting of both spatial (Morgan, Hansen \& Hillyard 1996) and nonspatial attention tasks (Painter, Dux, Travis \& Mattingley 2014; Muller et al 2006). Furthermore, the application of flicker SSVEPs in the face domain has revealed inversion effects at faster (15 and 20Hz), but not slower (5 and 10Hz) frequencies (Gruss, Wieser, 
Schweinberger, \& Keil, 2012). Whether face-selective effects at fast rates are modulated by familiarity remains unknown. Much remains to be learned about signatures of face processing at slower rates like $3.75 \mathrm{~Hz}$, possibly corresponding with higher-level visual processes, such as those indexed by the N250 and P300 ERP components. Additionally, it remains to be seen if different presentation frequencies can bias distinct visual processes without an explicit attentional manipulation, although it is clear that the EEG signal on the scalp changes with different presentation frequencies, suggesting different neural sources (Jacques et al 2016). If indeed a relationship exists between frequency and time domains as proposed by Rossion (2014), manipulation of stimulation frequency should enhance different stages of visual processing and we explore several of these in the current paradigm.

\section{Current Study}

The current study used a periodic visual presentation paradigm (flicker SSVEP) to investigate markers of familiar face recognition at different levels of visual processing. Faces were presented within streams of different frequencies $(15 \mathrm{~Hz}, 6 \mathrm{~Hz}$ and $3.75 \mathrm{~Hz})$ while participants completed an orthogonal task. Familiar and unfamiliar faces were presented in both upright and inverted configurations, with the overall expectation that familiarity effects would be evident for upright but not inverted faces, given abundant evidence showing that face recognition is compromised for inverted faces (Farah, Tanaka, \& Drain, 1995; Freire, Lee, Symons, 2000; Megreya \& Burton, 2006).

The fastest frequency $(15 \mathrm{~Hz})$, linked to early visual cortex responses (Muller et al., 2006; Robinson et al., 2017; Norcia et al. 2015), was likely to limit the extent of the representation within the face network. As such, we predicted that the $15 \mathrm{~Hz}$ signal would not yield any differences in the representation of familiar and unfamiliar faces. To the extent that any differences are observed, either in signal amplitude or using a multivariate analysis, these findings likely reflect low-level featural differences or perhaps differences in attention.

We predicted that familiarity effects would be observed at the "slower" frequencies, $6 \mathrm{~Hz}$ and $3.75 \mathrm{~Hz}$, which are associated with the $\mathrm{N} 170$ and N250 components of face processing, respectively. Thus, we predicted a reduction in the $6 \mathrm{~Hz}$ signal amplitude due to differential computation of visual features, perhaps mediated by the FFA, and that the responses to familiar and unfamiliar faces will be separable using a multivariate decoding approach.

Finally, we hypothesized that representations of familiar and unfamiliar faces would be distinct at $3.75 \mathrm{~Hz}$ frequency, with reduction in amplitude for familiar relative to unfamiliar faces, in accordance with previous ERP research (Zimmerman \& Eimer, 2013; Tanaka, Curran, Porterfield, \& Collins, 2006), with similar effects arising in the extended face network and likely associated with more identity or semantic information. Lastly, we predict that the separability of familiar and unfamiliar face responses using a multivariate decoding approach will correlate directly with behavioral familiarity, given ample evidence that familiarity effects are strongest in the N250 in the temporal domain (Henson, Mouchlianitis, Matthews, Kouider, 2008; Kaufmann, Schweinberger, \& Burton, 2009; Schweinberger, Pickering, Jentzsch, Burton, \& Kaufmann, 2002). 


\section{Materials and Methods}

\section{Participants}

This study was approved by the Institutional Review Board of Carnegie Mellon University (CMU). Informed consent was obtained from all participants. Participants were 20 adults, recruited from CMU (13 females). The experiment took roughly two hours and participants were compensated US $\$ 50$ for their time. One participant was excluded due to poor behavioral performance on the orthogonal color detection task, indicating lack of compliance. Participants completed the Edinburgh Handedness Inventory and a questionnaire regarding their age, gender race and ethnicity. Participants were Caucasian to avoid the potential influence of the other race face effect (Caldera et al., 2003; O'Toole, Deffenbacker, Valentin, \& Abdi, 1994). All participants were right-handed and had normal or corrected-to-normal vision.

\section{Design and Stimuli}

Experimental stimuli consisted of 100 face identities each of Western celebrities ("familiar" faces) and of Eastern European celebrities ("unfamiliar" faces; Figure 1). Forward facing images were obtained using Google image search. The task of equating familiar and unfamiliar image sets is notoriously difficult, but several steps were taken to equate them while still maintaining natural variation in the images. First, the faces were aligned at the eyes and cropped to an oval to minimize non-face features such as hair and clothing. Second, we used a very large image set (200 images) such that on average, low level image properties were likely similar across conditions. Third, we balanced gender across image sets, using 50 male and 50 female identities total. Fourth, we included 2 images per identity so that neural responses would be more likely to reflect identity processing rather than image-specific processing. Last, the unfamiliar faces were of European celebrities unknown to American participants and served as a matched unfamiliar face set.

On average, familiar and unfamiliar faces were very similar in terms of their low-level features (Figure 1b). To confirm the similarity of the image sets, the pixel-wise luminance of the familiar and unfamiliar face images were compared. T-tests conducted on the luminance value of each pixel revealed 18 pixels with significantly greater luminance for unfamiliar faces, and 2095 pixels with greater luminance for familiar faces $(p<.001$, uncorrected), out of approximately 102800 pixels per image, meaning only $2 \%$ of image pixels varied reliably between image sets. As can be seen in Figure 1c, pixels with the greatest luminance difference between familiar and unfamiliar faces were relatively distant from fixation and unlikely to be informative of face identity.

The stimuli were presented foveally within a rectangle $5 \times 6.87$ degrees of arc. Faces were presented upright or inverted 180 degrees. Blocks consisted of faces presented for 60 seconds of periodic visual presentation with $50 \%$ on/off cycles at either $3.75 \mathrm{~Hz}, 6 \mathrm{~Hz}$ or 15 $\mathrm{Hz}$. There were therefore three experimental factors: familiarity (familiar, unfamiliar), orientation (upright, inverted) and presentation frequency $(3.75,6$, and $15 \mathrm{~Hz})$. Both familiarity and orientation were blocked in this $2 \times 2 \times 3$ design. For clarification, we use the term 'block' to describe 60 second stimulation periods used during data collection. We will 
use the term 'trial' for the 4 second epochs that are derived from dividing blocks into smaller units for analysis.

During each block, faces were randomly chosen from the set of 100 familiar or unfamiliar face images and presented one at a time in a square wave fashion. Each block therefore included many faces (although of course this varied depending on presentation frequency). Participants performed a simple orthogonal task, namely to discriminate the number of times a face appeared with a green tint (1, 2 or 3 times) and respond using a key press. No feedback was given regarding accuracy. Note that each green face identity was consistent with the block condition (familiar or unfamiliar) and its duration was consistent with the presentation frequency, likely resulting in a slightly harder task in $15 \mathrm{~Hz}$ blocks compared to slower blocks. The green images were very infrequent and thus unlikely to influence neural processing in the frequency domain.

The Psychophysics Toolbox in Matlab was used to present the visual stimuli on a 24-inch LCD monitor with $60 \mathrm{~Hz}$ refresh rate. There were four 60 second blocks for each cell in our familiarity $(2) \times$ orientation $(2) \times$ frequency (3) design, resulting in 48 blocks for the whole experiment. Participants were offered small breaks between blocks. Previous findings show sufficient power exists to detect differences at this level of sampling (Nemrodov, Jacques, \& Rossion, 2015; Retter \& Rossion, 2017; Rossion \& Boremanse, 2011).

\section{Familiarity ratings}

To determine face familiarity for each participant individually, at the end of the experiment, participants were asked to rate their pre-experiment familiarity of each stimulus in the set. Each face was presented individually on the computer monitor and participants rated its familiarity on a scale from 1-7. Face order was randomized. Responses were made using key presses.

\section{EEG recording}

Continuous EEG data were recorded using a BioSemi Active Two system (BioSemi, Amsterdam, Netherlands), digitized at a 512-Hz sample rate with 24-bit A/D conversion. The 128 electrodes were arranged according to the international standard 10-5 system for electrode placement (Oostenveld \& Praamstra, 2001) using a nylon head cap. Online EEG was recorded using an active feedback circuit with the standard BioSemi Common Mode Sense (CMS) and Driven Right Leg (DRL) electrodes and no reference.

\section{EEG preprocessing}

EEG data analysis was performed offline using EEGLAB (Delorme \& Makeig, 2004). For preprocessing, bad electrodes were interpolated ( $N=1.21$ per participant) and all data were rereferenced to the common average of 128 electrodes. A high-pass $(0.1 \mathrm{~Hz})$ and low-pass $(100 \mathrm{~Hz})$ zero-phase filter, and $60 \mathrm{~Hz}$ notch filter were then applied using the standard EEGLAB filters. No trials were excluded due to artifacts, as these do not affect SNR in frequency space to the degree that they do in the time domain. This is standard practice for EEG frequency-domain analyses (Rossion, 2014). Additionally, our decoding approach is extremely adept at dealing with artifacts such as problematic trials (Grootswagers et al., 
2017). For each 60 second block, the first two and last two seconds of data were discarded, and the remaining 56 second block was split into $14 \times$ four second epochs. Each epoch consisted of 60, 24 or 15 cycles (images) for the 15,6 , or $3.75 \mathrm{~Hz}$ frequencies, respectively. Splitting blocks into a smaller number of epochs is a technique used to allow sufficient trial numbers for the decoding analysis. The 56 epochs per condition were reduced to 28 'pseudotrials' by taking the mean of every two epochs to obtain a characteristic time course. The pseudotrial averaging technique was chosen for two reasons: (1) it allowed a more accurate representation of the periodic signal by reducing non-stimulus related EEG noise (Yeatman \& Norcia, 2016), and (2) averaging also improves classification accuracy (Grootswagers, Wardle, \& Carlson, 2017). A discrete Fourier transform was applied to the pseudotrials using the FFT function in MATLAB, normalized for the 2048 time points $(512 \mathrm{~Hz}$ sampling frequency $\times 4$ seconds/epoch).

\section{Univariate FFT analysis}

For each condition and pseudotrial per participant, a signal-to-noise (SNR) baseline correction was applied to the FFT data using the Letswave 6 toolbox (http:// nocions.webnode.com/letswave). SNR was calculated as the ratio between the amplitude of the relevant frequency bin and the mean of its 10 surrounding frequency bins, excluding the two neighboring bins and the most extreme bin, a typical baseline correction technique (Rossion et al 2012). The mean SNR was then taken across all pseudotrials. The SNR at the corresponding frequency $(15 \mathrm{~Hz}, 6 \mathrm{~Hz}$ or $3.75 \mathrm{~Hz}$, depending on the image presentation rate) was analyzed.

\section{Decoding analysis}

To assess whether there might be subtle changes in the spatial distributions of evoked signal across electrodes that were not obvious in the gross univariate analyses, we used a classifier to decode between patterns of activity evoked by familiar faces relative to unfamiliar faces (familiarity decoding) and in a separate analysis, decode between upright and inverted faces (orientation decoding). A support vector machine (SVM) classifier was used to decode the neural activity evoked by the different stimuli types. Depending on the stimulus presentation frequency, the amplitude at $15 \mathrm{~Hz}, 6 \mathrm{~Hz}$, or $3.75 \mathrm{~Hz}$ was selected for each electrode. The FFT amplitudes for each electrode were used as features for classification. Data for each electrode were z-scored across all trials to standardize electrodes before classification.

For all analyses, two-way classification was performed to decode between familiarity (familiar/unfamiliar) or face orientation (upright/inverted) for all levels of the other factors. Fourfold cross-validation was performed such that the training data per class for each fold were pseudotrials from 3 of the trimmed 56-second blocks ( 21 pseudotrials) and test data were pseudotrials from the remaining original 56-second block (7 pseudotrials). This crossvalidation approach reduced the likelihood that correct classification would be inflated due to any inherent correlation between pseudotrials within the same block; in other words, the classifier had to generalize to different blocks.

Searchlight decoding.-A searchlight decoding approach was used to elucidate clusters of electrodes that resulted in the best classification accuracy. This data driven approach 
allowed us to determine the topographic characteristics of the neural correlates of face familiarity and orientation at the different presentation frequencies. Although the focus was primarily on the familiar versus unfamiliar distinction, because the face inversion effect is so well-established, we include this here as a further benchmark to show the validity of the effects we obtain. That is, the orientation decoding analysis had the added advantage of allowing us to compare the effect of face inversion for familiar faces to that of unfamiliar faces.

For each of the 128 electrodes, all the surrounding electrodes within a distance of $60 \mathrm{~mm}$ were calculated using the Fieldtrip toolbox (Maris and Oostenveld 2007), resulting in 128 clusters (median $=20$ electrodes per cluster, $\min =8, \max =23$ ). Two-way classification was performed using each of these clusters and classification accuracy was averaged across participants.

Whole brain decoding.-To compare classification accuracy across classes (e.g., familiarity decoding for upright versus inverted faces) within a given frequency, classification was performed using all 128 electrodes as features. Decoding accuracy was then assessed as a function of the face familiarity ratings from each participant (mean ratings for familiar and unfamiliar faces). We hypothesized that participants for whom the famous faces were rated as more familiar might have better separability between conditions and therefore more accurate decoding, particularly for the familiarity decoding analysis.

\section{Cluster-based permutation testing}

For both the univariate and multivariate results, significance was assessed using clusterbased permutation testing as implemented in the Fieldtrip toolbox (Maris \& Oostenvald, 2007). Cluster-based statistics were performed for all relevant pairwise comparisons

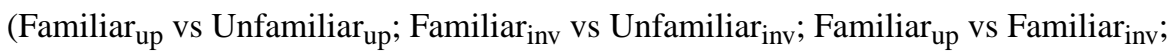
Unfamiliar $_{\text {up }}$ vs Unfamiliar ${ }_{\text {inv }}$ ). For each comparison, electrodes varying at the group level using an alpha level of .05 were considered in the cluster-based analysis, and clusters of neighboring candidate electrodes $(\mathrm{min}=2)$ were assessed for significance using 1000 permutations and comparing the cluster-level statistic (sum of $t$-values) to the null distribution. Cluster significance was assessed using two-tailed alpha $=.05$ (alpha $=.025$ per tail). In addition to the pairwise comparisons, the univariate SNR was statistically compared with 1 (noise level, which indicates that surrounding frequency bins had the same amplitude as the stimulation frequency) and the searchlight decoding results were statistically compared to 50\% (chance decoding) to assess significance of the signals. For all presented results, the $p$-value from a cluster-based permutation test refers to significance of a single significant or near significant cluster that was identified using this method.

\section{Results \\ Color task}

On the orthogonal task, designed to ensure that participants were fixating and attending to the center of the screen, participants correctly identified the number of green faces well above chance. Accuracy was $87.50 \%$ in the $15 \mathrm{~Hz}, 95.39 \%$ in the $6 \mathrm{~Hz}$ and $92.76 \%$ in the 
$3.75 \mathrm{~Hz}$ image presentation trials. Within each of the presentation frequencies, there was no significant effect of face familiarity or orientation on accuracy of color change detection, $F S$ $<4.17$, ps $>.056$. The findings demonstrate that participants were engaged in the orthogonal color detection task. Unsurprisingly, the $15 \mathrm{~Hz}$ blocks were slightly more challenging than slower frequency presentation blocks, though non-significantly.

\section{Familiarity ratings}

After completion of the EEG procedure, each participant completed familiarity ratings of every stimulus in the image set. On a scale from 1-7, participants rated the "familiar" famous faces ( $M=4.77, S D=1.26$ ) to be more familiar than the "unfamiliar" faces ( $\mathrm{M}=$ $1.97, S D=.82), t_{18}=8.03, p<.001$, indicating that the familiarity manipulation was successful. Although some participants may have developed familiarity with the unknown faces over the course of the experiment (albeit without any obvious semantic associations), the data show clear evidence that familiarity was significantly different between the two stimulus types.

For each participant, we calculated the difference between the familiarity ratings for familiar and unfamiliar faces. Participants were classified as having "high" familiarity with the experimental faces or "low" familiarity based on a median split of the difference scores (median = 3.16; "high" $M=4.00$, "low" $M=1.56$ ). We consider the profile of familiarity rating below in conjunction with the EEG data.

\section{Topography of responses}

Fourier analyses revealed clear periodic EEG signal at the frequency of image presentation. As can be seen in Figure 2a, the SNR frequency spectra revealed peaks at the fundamental frequency and its harmonics for the $15 \mathrm{~Hz}, 6 \mathrm{~Hz}$ and $3.75 \mathrm{~Hz}$ image presentation frequencies. EEG responses were strongest over occipito-temporal brain regions, but the spatial extent of the signal differed markedly depending on the presentation frequency (Figure 2b). Cluster-based permutation testing confirmed that SNR was significantly above 1 across the whole brain for all levels of frequency, familiarity and orientation, cluster $t s>$ 503.65, $p$ s <.001. The $15 \mathrm{~Hz}$ signal was strongest over mid-occipital regions, with the largest signal at electrode $\mathrm{Oz}$, in line with previous studies, which have linked this presentation frequency to early visual cortex responses (Muller et al., 2006; Robinson et al., 2017). In contrast, the $6 \mathrm{~Hz}$ and $3.75 \mathrm{~Hz}$ signals were consistent with lateral occipitotemporal responses. Visual inspection of the topographic distribution revealed that greater right than left lateralized responses were particularly evident for the $6 \mathrm{~Hz}$ frequency (largest signal at PO8). SNR for the $3.75 \mathrm{~Hz}$ frequency was largest at PO8 and there was a secondary cluster around PO7. These differences in topography suggest that the frequency manipulation was successful in tapping into different levels of face processing.

Due to the differences in the hypothesized results, as well as differences in topography, we analyzed the results from each presentation frequency separately. Also, because the $15 \mathrm{~Hz}$ condition had considerably more cycles (60) than the $6 \mathrm{~Hz}$ (24 cycles) and $3.75 \mathrm{~Hz}$ (15 cycles) conditions, including these in the same analysis is ill-advised. 
To investigate the effect of face familiarity and orientation on the periodic signal, for each frequency, we conducted cluster-based permutation tests on the flicker SSVEP, comparing both familiar versus unfamiliar faces, and upright versus inverted faces. Next, we detail the searchlight decoding results for the same comparisons, and finally we summarize results of a whole brain multivariate decoding analysis for the familiarity and orientation comparisons. Given the large number of analyses and comparisons, we provide Table 1 as a summary of all the findings from both the univariate and multivariate analyses.

\section{Univariate results}

For the relevant pairwise comparisons (Familiar ${ }_{\text {up }}{\text { vs } \text { Unfamiliar }_{\text {up }} \text {; Familiar }}_{\text {inv }}$ vs Unfamiliar $_{\text {inv }}$ ), cluster-based permutation tests were used to compare the strength of the neural signal (SNR) at the presentation frequency.

(a.) Analysis at $15 \mathrm{~Hz}$-As can be seen in Figure 3 and in Table 1, upright familiar faces had significantly stronger signal than upright unfamiliar faces, and this effect was particularly pronounced over central occipital regions, cluster $t=163.42, p=.002$. There was no significant difference between inverted familiar and inverted unfamiliar faces, $p>$. 050. Taken together, the $15 \mathrm{~Hz}$ results indicate that a familiarity effect is evident for upright, but not inverted faces.

(b.) Analysis at $\mathbf{6 H z}$ - In contrast to the $15 \mathrm{~Hz}$ results, cluster-based permutation tests revealed that upright familiar faces had marginally lower signal at $6 \mathrm{~Hz}$ than upright unfamiliar faces, particularly over right occipito-temporal regions, cluster $t=-28.71, p=$. 055 (Figure 3; Table 1). Again, there was no significant difference between inverted familiar versus unfamiliar faces, cluster $p>.05$. These results indicate that familiar faces have a distinct neural signature at $6 \mathrm{~Hz}$ that is qualitatively different from that at $15 \mathrm{~Hz}$.

(c.) Analysis at $3.75 \mathrm{~Hz}$-The responses to faces presented at $3.75 \mathrm{~Hz}$ revealed no significant differences between familiar and unfamiliar faces, whether they were upright or inverted, $p \mathrm{~s}>.05$ (Figure 3; Table 1). This is in stark contrast to the results at the faster frequencies.

In summary, the univariate analysis of frequency tagged responses elicited by familiar and unfamiliar faces revealed differences in face processing that varied by the stimulation frequency. Notably, familiar faces had stronger signal at $15 \mathrm{~Hz}$ and marginally reduced signal at $6 \mathrm{~Hz}$ relative to unfamiliar faces and there was no difference at $3.75 \mathrm{~Hz}$. Importantly, when present, the familiar versus unfamiliar comparisons yielded significant effects when the faces were upright, but not inverted, indicating that the familiar/unfamiliar differences were unlikely to be driven by low-level stimulus differences.

\section{Multivariate Decoding Analysis}

(a.) Analysis at $15 \mathrm{~Hz}$-Figure 4 shows all multivariate results for the $15 \mathrm{~Hz}$ frequency. Searchlight decoding results for familiar versus unfamiliar faces revealed significant decoding at $15 \mathrm{~Hz}$ for upright faces, cluster $t=64.62, p=.010$, and for inverted faces, cluster $t=57.41, p=.018$. In a direct comparison of upright and inverted conditions, a 
cluster corrected permutation test revealed no significant differences between decoding for upright versus inverted faces, $p=.157$. To establish if behavioral familiarity predicted decoding performance, the same familiarity decoding approach was performed on the whole brain data (all 128 electrodes). We assigned participants as having "high" or "low" familiarity with the experimental faces (see Methods). A $2 \times 2$ ANOVA was conducted on the whole brain familiarity decoding results with factors of orientation (upright or inverted faces) and behavioral familiarity (high or low familiarity participants). This test revealed no significant main effects and no significant interaction, $F s<1.22$, ps $>.287, \eta_{\mathrm{G}}^{2}<.035$. Overall, the $15 \mathrm{~Hz}$ analyses reveal no substantial modulations of responses by familiarity, other than a univariate amplitude difference, which may be potentially explained by the small $2 \%$ difference in the image statistics. Findings from familiarity analyses are summarized in Table 1.

In a secondary analysis, we tested for differences in decoding orientation, separately, for familiar and unfamiliar conditions. Classification was performed between upright and inverted faces. Using the searchlight approach, there was significant orientation decoding of both familiar and unfamiliar faces, $p s<.001$, and this was most pronounced over central occipital electrodes. There was no significant difference in orientation decoding for familiar and unfamiliar faces, $p>.050$. The whole brain decoding echoed these results, with significant orientation decoding for both familiar and unfamiliar faces, $t s>5.23, p s<.001$. A 2-way ANOVA with repeated measures variable of familiarity and between measures variable of behavioral familiarity (difference between familiar and unfamiliar face ratings, based on a median split) revealed a marginal effect of face familiarity, $F_{1,16}=3.67, p=.073$, no significant main effect of behavioral familiarity, $F_{1,16}=2.82, p=.112$, and no interaction, $F_{1,16}<1, p=.978$. Both upright and inverted faces therefore exhibited distinct and robust neural signatures at $15 \mathrm{~Hz}$.

(b.) Analysis at $6 \mathrm{~Hz}-$ For the familiarity decoding analysis using the searchlight method (see Figure 5), there was significant decoding for upright faces, $p<.001$, with the largest decoding accuracy for a cluster around electrode P4 $(M=59.77 \%)$. There was also significant, albeit lower, classification for inverted faces, $p=.023$, with maximum decoding centered at P10 $(\mathrm{M}=55.73 \%)$. Furthermore, familiarity decoding was greater for upright than inverted faces, but this did not quite reach significance at the cluster level, $p=.081$. Using whole brain decoding to ascertain the effect of familiarity across orientation, a $2 \times 2$ ANOVA revealed a significant main effect of face orientation, $F_{1,16}=11.26, p=.004, \eta_{\mathrm{G}}^{2}$ $=.157$, such that decoding was higher for upright than inverted faces. There was no significant main effect of participant familiarity rating. There was a significant interaction, $F$ $=4.79, p=.044, \eta_{\mathrm{G}}^{2}=.073$, but follow up tests revealed no significant differences between high and low familiarity participants for upright $\left(t_{16}=-1.30, p=.212\right)$ or for inverted faces $\left(t_{16}=.964, p=.350\right)$.

There was also significant orientation decoding for both familiar and unfamiliar faces using the searchlight approach ( $p s<.001$, see Figure 5), but no significant difference between familiar and unfamiliar faces, $p=.149$. This was surprising given the striking difference in the number of electrodes on which significant decoding was observed (Figure 5C) for upright relative to inverted conditions. For the orientation decoding analysis using all 128 
channels, again decoding was significant for both familiar and unfamiliar faces, but there were no differences across face familiarity or behavioral familiarity, $F_{S}<1.56, p s>.230$, $\eta_{\mathrm{G}}^{2}<.081$.

Overall, these results show that distinct neural signatures for familiar and unfamiliar faces were evident at $6 \mathrm{~Hz}$, but only when the faces were upright. Effects of orientation were present for both familiar and unfamiliar faces, but with only marginally significant differences between them.

(c.) Analysis at 3.75-The familiarity decoding results at $3.75 \mathrm{~Hz}$ revealed a substantially different pattern (Figure 6). Searchlight analyses revealed significant familiarity decoding for upright faces $(p=.007)$, with maximal decoding over left occipitotemporal regions ( $M=59.12$, electrodes P5 and PP05h). There was no significant decoding for inverted faces $(p=.140)$. However, a cluster corrected permutation test revealed no differences in decoding between familiarity conditions $(p>.05)$. Whole brain decoding revealed a significant main effect of behavioral familiarity, $F_{1,16}=6.74, p=.020$, $\eta_{\mathrm{G}}^{2}=0.164$. There was also a marginal interaction between behavioral familiarity ratings and orientation for familiarity decoding, $F_{1,16}=3.57, p=.077, \eta_{\mathrm{G}}^{2}=.106$, such that familiarity decoding for upright faces was better for participants with "high" familiarity than those with "low" familiarity, $t_{16}=2.70, p=.016$. There was no difference in familiarity decoding between these participants for inverted faces, $t_{16}=.49, p=.628$. Using a second approach to confirm the presence of this effect, a linear mixed effects analysis on the 3.75 $\mathrm{Hz}$ decoding results with fixed effects of orientation and behavioral familiarity scores similarly revealed a significant effect of behavioral familiarity, $F_{1,17}=5.14, p=.037$, such that larger familiarity scores were associated with higher classification between familiar and unfamiliar faces.

Orientation decoding for the $3.75 \mathrm{~Hz}$ presentation rate revealed significantly above chance decoding for both familiar and unfamiliar faces $(p s<.001)$. Again, although decoding seemed to be reliable at greater number of individual electrodes for unfamiliar faces, there was ultimately no difference between orientation decoding for familiar and unfamiliar faces $(p>.05)$. For the whole brain analysis, a $2 \times 2$ ANOVA of face familiarity and behavioral familiarity scores revealed a significant main effect of behavioral familiarity such that participants with higher familiarity had better orientation decoding, $F_{1,16}=6.54, p=.021$, $\eta_{\mathrm{G}}^{2}=.233$. There were no significant main effects of familiarity and no significant interaction, $p s>.626$. Again, a linear mixed effects model revealed a significant effect of behavioral familiarity on decoding, $F_{1,17}=7.51, p=.014$, such that higher familiarity led to better face orientation decoding.

Overall, the results at $3.75 \mathrm{~Hz}$ revealed distinct patterns of neural activity in response to the familiar and unfamiliar faces for participants that were most familiar with the experimental faces. That is, $3.75 \mathrm{~Hz}$ responses were more distinct for those participants who most familiar with the face identities in the "familiar" image set. Individual familiarity also influenced orientation decoding, suggesting that this frequency might tap into higher-level cognitive processes. The results from each statistical analysis at each presentation frequency are reviewed in Table 1. 


\section{Discussion}

The aim of the current study was to explore the extent to which distinct temporal patterns of neural activity emerge during the recognition of familiar and unfamiliar faces. Using a highpowered periodic visual stimulation paradigm (flicker SSVEP) at three frequencies (15, 6, and $3.75 \mathrm{~Hz}$ ), while concurrently recording scalp EEG, we showed that familiar and unfamiliar faces evinced distinct temporal processes at multiple frequencies. Furthermore, our searchlight decoding methods enabled us to characterize subtle changes in the patterns of electrophysiological activity across conditions, offering novel insights into the nature of representations mediating face perception.

The first key finding, as evident from the topographies (Figure 2) and the profile of the signals, indicates that the three different frequencies of stimulus presentation evoked qualitatively distinct distributions of evoked power, replicating previous findings (Jacques et al 2016). While precise estimates of source generators cannot be made in the absence of high-quality source localization analysis, we offer speculations concerning which cortical regions might be implicated at the different frequencies. The $15 \mathrm{~Hz}$ signal, the fastest frequency, clearly drove responses most strongly over the most posterior central electrodes, perhaps reflecting activity in early visual cortex. In contrast, the $6 \mathrm{~Hz}$ presentations evoked bilateral posterior responses, with a bias toward the right hemisphere, perhaps implicating occipito-temporal regions such as the fusiform face area. The $3.75 \mathrm{~Hz}$ response also exhibited this posterior bilateral spatial pattern, but substantial activity was also evident in anterior electrode regions, perhaps implicating processing within the extended face network. These findings offer insights into the different temporal dynamics and spatial arrangements evoked by different frequencies.

In the univariate analyses, at $15 \mathrm{~Hz}$, upright familiar faces evoked greater power than upright unfamiliar faces over central posterior electrodes, but this difference was not present when faces were inverted. A closer look at the activity pattern itself showed that there was no significant difference in familiarity (familiar/unfamiliar) decoding when faces were upright compared to inverted, or in orientation (upright/inverted) decoding in familiar and unfamiliar face conditions. Decoding orientation, however, was possible within both familiar and unfamiliar face conditions. This suggests no difference in the spatial distribution of familiar and unfamiliar face responses, rather, the results point to a simple change in amplitude of the signal. Greater amplitude responses to familiar than to unfamiliar faces may reflect the classic effect of attention and with previous SSVEP studies showing that spatial and nonspatial attention enhances SNR (Muller et al., 2006; Norcia et al. 2015). However, we cannot definitively establish attention as the mechanism driving this result, because we did not explicitly control and measure all aspects of attention in our paradigm. In fact, we observed a small difference in less than $2 \%$ of pixels between averaged stimuli of each condition, suggesting luminance differences alone may be responsible for this effect. If, however, attention is driving the increased response to familiar faces, we speculate that the difference in activity may reflect contributions from luminance sensitive neurons in early visual cortex. This mechanism could account for the qualitative differences between $15 \mathrm{~Hz}$ and the slower $6 \mathrm{~Hz}$ and $3.75 \mathrm{~Hz}$ conditions, suggesting effects from slower frequencies do not strictly reflect attention. Although presenting faces at $15 \mathrm{~Hz}$ likely precluded individuation of all face 
identities, participants may have recognized a few faces over the entire 1-minute block, thereby enhancing attention to the stream of familiar, but not unfamiliar faces. Consistent with this interpretation, previous SSVEP research has shown that participants elicit differentiated responses when detecting certain higher-level visual facial features such as expression (Wieser \& Keil, 2014) or gaze (Wieser, Miskovic, Rausch, \& Keil, 2014) at fast presentation frequencies.

In contrast, at $6 \mathrm{~Hz}$ presentation rate, familiar and unfamiliar face responses were successfully decoded, with more widespread decoding for upright than inverted faces. There was no relationship, however, between behavioral familiarity ratings and familiarity decoding performance. Although there were no significant differences between familiarity conditions, orientation was also successfully decoded within familiar and unfamiliar face conditions. Importantly, the univariate analysis showed that familiar faces evoked less power compared to unfamiliar faces at $6 \mathrm{~Hz}$, possibly reflecting an adaptation response. This also suggests a qualitative separation between "fast" and "slow" processes that uniquely subserve familiar face recognition. At $6 \mathrm{~Hz}$ presentation rate, faces were presented once about every $170 \mathrm{~ms}$. As proposed by Rossion (2014), $6 \mathrm{~Hz}$ power evoked by face presented at the same rate may reflect an aggregate of the same process as the N170 from individual faces. However, the N170 is not generally modulated by familiarity, and instead, likely reflects structural encoding phases of face recognition. The results here may be inconsistent with this hypothesis since familiarity is successfully decoded when faces are presented at $6 \mathrm{~Hz}$. Alternatively, the frequency tagging approach used here and more extensively by Rossion (2014) may have more power to detect subtle differences in face processing compared to traditional ERP approaches and the multivariate analytics might have contributed as well. Additionally, familiar face recognition might engage pattern recognition involving the cooccurrence of certain visual features in an orientation-invariant manner. This is quite likely to be an independent (or at least separable) effect from the cognitive aspects of recognition, because we know that recognition is disrupted when faces are inverted (Farah, Tanaka, \& Drain, 1995). It seems plausible that learning an individual's face might specifically involve pattern recognition processes within FFA, in a similar way expertise influences activity in this region (Gauthier et al., 1999).

Finally, when viewing faces at $3.75 \mathrm{~Hz}$, there was no modulation of amplitude in our univariate analysis, different from what we predicted. However, familiarity decoding was possible for upright (strongest in the left and central posterior electrodes), but not inverted faces. The difference between upright and inverted conditions did not quite reach significance, despite the availability of longer processing time per image than the $6 \mathrm{~Hz}$ condition. Our analysis with familiarity ratings clearly shows an effect of pre-experiment familiarity with familiarity decoding at $3.75 \mathrm{~Hz}$. That is, individual participants who reported greater differences in familiarity between familiar and unfamiliar face identities, evinced better decoding than individuals who reported more similar familiarity ratings between stimulus groups. Interestingly, this interaction is present only when faces are presented at $3.75 \mathrm{~Hz}$, and not at faster frequencies. This may also explain why we no group differences in univariate amplitude comparisons were observed. That is, the signal evoked by $3.75 \mathrm{~Hz}$ presentation may simply be most sensitive to individual differences in behavior. The presence of this effect in the slowest frequency dovetails neatly with recent findings from 
familiar face recognition in the primate visual system showing that two regions in the anterior temporal lobe (within the extended face processing network) exhibit a non-linear response to accumulating high frequency information in familiar face recognition (Landi \& Freiwald, 2017). That is, as high spatial frequency information is added to a familiar, but not unfamiliar, face stimulus, only these two anterior temporal regions, and not more posterior regions, evince a sudden burst of activity once sufficient visual information is present, akin to sudden recognition of a familiar face. A similar finding was documented in the human temporal lobe and amygdala (Ramon et al., 2015). Because these studies were conducted in the spatial domain (fMRI), it is difficult to make inferences in the temporal domain. Our results offer complementary insights into the temporal nature of this spatial non-linearity. That familiarity ratings correlated with decoding performance at the slowest presentation frequency, but not faster ones, despite significant decoding in $3.75 \mathrm{~Hz}, 6 \mathrm{~Hz}$ and $15 \mathrm{~Hz}$ frequencies, suggests there may be a non-linear relationship between processes that are underway within the first $260 \mathrm{~ms}(3.75 \mathrm{~Hz})$ compared to those instigated during the first $170 \mathrm{~ms}(6 \mathrm{~Hz})$.

Though the results here suggest a three-way dissociation between patterns of activity evoked by different presentation frequencies, an interpretational caveat remains. In the current paradigm, each sequential face stimulus essentially masks the one before it, presumably interrupting processing of the prior stimulus. Some suggest that SSVEPs reflect a linear superposition of individual evoked potentials (Capilla et al., 2011), but evidence exists that nonlinear effects contribute to the evoked responses measured in the frequency domain (Liu et al., 2010). It is not clear to what extent top-down, or other intermediary processes continue after the next face is displayed. It is also important to note that neural processing of periodic images is not completely limited to the time of image presentation. Studies of rapid serial visual presentations (RSVP) have shown that it is possible to detect and discriminate certain targets during a visual stream, meaning that stimuli appearing for short durations can undergo high level processing, but that there are clear limitations to processing more than one stimulus in quick succession (e.g., the attentional blink: Shapiro, Raymond \& Arnell, 1997). A recent study showed that all images presented using RSVP could be reliably classified within early time windows ( $<150 \mathrm{~ms}$ ), and activations of images temporally close to a target were sustained for longer periods of time, but that only targets elicited late selection activation (>300 ms) (Marti \& Dehaene 2017). Therefore, periodic EEG responses, such as those observed here, might be influenced by selection of images for further processing and we cannot be certain about the degree to which an evoked response reflects activity occurring selectively within a given timeframe, or frequency. This may explain why familiarity appears to modulate $15 \mathrm{~Hz}$ responses, which predominate in electrodes over earlier visual cortex. This problem is not necessarily specific to frequency tagging, as the same challenge may be present in traditional ERP designs where stimuli are also presented sequentially, but typically at a slower rate.

There were also clear differences in hemispheric laterality in our findings. In both $6 \mathrm{~Hz}$ and $3.75 \mathrm{~Hz}$ presentation frequencies, differences in the neural patterns evoked during familiar and unfamiliar face recognition were evident over bilateral occipitotemporal electrodes, despite stronger responses over the right hemisphere for all faces. These results are consistent with other studies of familiar face recognition. Pourtois et al. (2005) found that 
repetition of familiar faces across different viewpoints reduced BOLD activation in left middle temporal and inferior frontal cortex activity, while unfamiliar faces evinced the same effect, but only in the right hemisphere. The engagement of the left hemisphere may result from access to lexical or semantic information from the visual modality. Both proper-names and famous faces activate the left middle temporal gyrus more than common names (GornoTempini et al. 1998) and lesions to left temporal-occipital lesions may be a result of a semantic impediment (Gainotti \& Marra, 2011). Consistently, an ERP study which used famous faces and an explicit naming task found broad recruitment of left hemisphere (Gosling \& Eimer, 2011). Taken together, these findings suggest that the current left and right hemisphere topography for familiar face decoding found reflect the contribution of visual and language/semantic processes.

Although the current study successfully distinguished between familiar and unfamiliar face recognition at multiple frequency stimulations, significant questions regarding the nature of familiar face processing remain. Familiar face recognition arises through experience. That is, novel faces are initially unfamiliar, but with some experience, become familiar. The neural processes which subserve this representational evolution or the microgenesis to familiarity remain unknown. Additionally, the current study used a large group of familiar and unfamiliar face identities, paired with an orthogonal task, rather than a small group of faces paired with the task of individuation. The results reported here, then, reflect large-scale differences along the visual hierarchy between familiar and unfamiliar face processing, rather than more naturalistic individuation during which representations of a single face unfold over time.

In conclusion, the current study explored differences in temporal processes recruited during familiar and unfamiliar face processing. Our approach utilized a combination of flicker SSVEP paradigm and searchlight decoding analysis of EEG data, representing a methodological advance over traditional ERP approaches. Using these methods, we showed a dissociation between responses to familiar and unfamiliar faces evoked at three presentation frequencies. These dissociations show that familiar and unfamiliar face recognition recruit distinct neural resources with distinct spatial distributions within multiple frequency streams, putatively linked to distinct contributions to face recognition.

\section{Acknowledgements:}

This work was supported by a National Science Foundation grant to M.B. (BCS-1354350). We thank Jieming Li for assistance in data collection, and Dr. Tijl Grootswagers for helpful suggestions on the decoding analysis.

\section{References}

Alonso-Prieto E, Van Belle G, Liu-Shuang J, Norcia AM, \& Rossion B (2013). The 6Hz fundamental stimulation frequency rate for individual face discrimination in the right occipito-temporal cortex. Neuropsychologia, 51(13), 2863-2875. [PubMed: 24007879]

Althoff RR, \& Cohen NJ (1999). Eye-movement-based memory effect: a reprocessing effect in face perception. J Exp Psychol Learn Mem Cogn, 25(4), 997-1010. [PubMed: 10439505]

Barense MD, Henson RN, \& Graham KS (2011). Perception and conception: temporal lobe activity during complex discriminations of familiar and novel faces and objects. J Cogn Neurosci, 23(10), 3052-3067. doi:10.1162/jocn_a_00010 [PubMed: 21391761] 
Barton JJS, Radcliffe N, Cherkasova MV, Edelman J, \& Intriligator JM (2006). Information processing during face recognition: the effects of familiarity, inversion, and morphing on scanning fixations. Perception, 35(8), 1089-1105. [PubMed: 17076068]

Bruce V, Henderson Z, Newman C, \& Burton AM (2001). Matching identities of familiar and unfamiliar faces caught on CCTV images. J Exp Psychol Appl, 7(3), 207-218. [PubMed: 11676099]

Burton AM (2013). Whyhas research in face recognition progressed so slowly? The importance of variability. The Quarterly Journal of Experimental Psychology, 66(1467-1485).

Caldara R, Thut G, Servoir P, Michel CM, Bovet P, \& Renault B (2003). Face versus non-face object perception and the 'other-race'effect: a spatio-temporal event-related potential study. Clinical Neurophysiology, 114(3), 515-528. [PubMed: 12705432]

Campanella S, Bourguignon M, Peigneux P, Metens T, Nouali M, Goldman S, ... De Tiege X (2013). BOLD response to deviant face detection informed by $\mathrm{P} 300$ event-related potential parameters: a simultaneous ERP-fMRI study. Neuroimage, 71, 92-103. doi:10.1016/j.neuroimage.2012.12.077 [PubMed: 23313569]

Davies-Thompson J, Gouws A, \& Andrews TJ (2009). An image-dependent representation of familiar and unfamiliar faces in the human ventral stream. Neuropsychologia, 47(6), 1627-1635. doi: 10.1016/j.neuropsychologia.2009.01.017 [PubMed: 19428408]

Delorme A, \& Makeig S (2004). EEGLAB: an open source toolbox for analysis of single-trial EEG dynamics including independent component analysis. Journal of neuroscience methods, 134(1), 921. [PubMed: 15102499]

Di Russo F, Pitzalis S, Aprile T, Spitoni G, Patria F, Stella A, ... \& Hillyard SA (2007). Spatiotemporal analysis of the cortical sources of the steady-state visual evoked potential. Human brain mapping, 28(4), 323-334. [PubMed: 16779799]

Dubois S, Rossion B, Schiltz C, Bodart JM, Michel C, Bruyer R, et al. (1999). Effect of familiarity on the processing of human faces. Neuroimage, 9, 278-289. [PubMed: 10075898]

Eimer M (2000). Event-related brain potentials distinguish processing stages involved in face perception and recognition. Clinical neurophysiology, 111(4), 694-705. [PubMed: 10727921]

Ewbank MP, \& Andrews TJ (2008). Differential sensitivity for viewpoint between familiar and unfamiliar faces in human visual cortex. Neuroimage, 40(4), 1857-1870. doi:10.1016/ j.neuroimage.2008.01.049 [PubMed: 18343161]

Farah MJ, Tanaka JW, \& Drain HM (1995). What causes the face inversion effect? Journal of Experimental Psychology: Human perception and performance, 21(3), 628. [PubMed: 7790837]

Freire A, Lee K, \& Symons LA (2000). The face-inversion effect as a deficit in the encoding of configural information: Direct evidence. Perception, 29(2), 159-170. [PubMed: 10820599]

Freiwald W, Duchaine B, \& Yovel G (2016). Face Processing Systems: From Neurons to Real-World Social Perception. Annu Rev Neurosci, 39, 325-346. doi:10.1146/annurev-neuro-070815-013934 [PubMed: 27442071]

Gainotti G, \& Marra C (2011). Differential contribution of right and left temporo-occipital and anterior temporal lesions to face recognition disorders. Front Hum Neurosci, 5, 55. doi:10.3389/fnhum. 2011.00055 [PubMed: 21687793]

Gauthier I, Tarr MJ, Anderson AW, Skudlarski P, \& Gore JC (1999). Activation of the middle fusiform 'face area' increases with expertise in recognizing novel objects. Nature neuroscience, 2(6).

Gobbini MI, Gors JD, Halchenko YO, Rogers C, Guntupalli JS, Hughes H, \& Cipolli C (2013). Prioritized Detection of Personally Familiar Faces. PLoS One, 8(6), e66620. doi:10.1371/ journal.pone.0066620 [PubMed: 23805248]

Gobbini MI, \& Haxby JV (2007). Neural systems for recognition of familiar faces. Neuropsychologia, 45(1), 32-41. doi:10.1016/j.neuropsychologia.2006.04.015 [PubMed: 16797608]

Gold JM, Barker JD, Barr S, Bittner JL, Bratch A, Bromfield WD, ... Srinath A (2014). The perception of a familiar face is no more than the sum of its parts. Psychon Bull Rev, 21(6), 14651472. doi:10.3758/s13423-014-0632-3 [PubMed: 24777442]

Gorno-Tempini ML, Price CJ, Josephs O, Vandenberghe R, Cappa SF, Kapur N, .. \& \& Tempini ML (1998). The neural systems sustaining face and proper-name processing. Brain: a journal of neurology, 121 (11), 2103-2118. [PubMed: 9827770] 
Gosling A, \& Eimer M (2011). An event-related brain potential study of explicit face recognition. Neuropsychologia, 49(9), 2736-2745. [PubMed: 21679721]

Grootswagers T, Wardle SG, \& Carlson TA (2017). Decoding dynamic brain patterns from evoked responses: A tutorial on multivariate pattern analysis applied to time series neuroimaging data. Journal of cognitive neuroscience, 29 (4), 677-697, 10.1162/jocn_a_01068 [PubMed: 27779910]

Gruss LF, Wieser MJ, Schweinberger SR, \& Keil A (2012). Face-evoked steady-state visual potentials: effects of presentation rate and face inversion. Frontiers in Human Neuroscience, 6: 316. doi: 10.3389/fnhum.2012.00316 [PubMed: 23205009]

Guntupalli JS, Wheeler KG, \& Gobbini MI (2017). Disentangling the Representation of Identity from Head View Along the Human Face Processing Pathway. Cereb Cortex, 27(1), 46-53. doi:10.1093/ cercor/bhw344 [PubMed: 28051770]

Hanso L, Bachmann T, \& Murd C (2010). Tolerance of the ERP Signatures of Unfamiliar versus Familiar Face Perception to Spatial Quantization of Facial Images. Psychology, 1, 199-208.

Haxby JV, Hoffman EA, \& Gobbini MI (2000). The distributed human neural system for face perception. Trends in cognitive sciences, 4(6), 223-233. [PubMed: 10827445]

Henson RN, Mouchlianitis E, Matthews WJ, \& Kouider S (2008). Electrophysiological correlates of masked face priming. Neuroimage, 40(2), 884-895. [PubMed: 18234522]

Henson R, Shallice T, \& Dolan R (2000). Neuroimaging evidence for dissociable forms of repetition priming. Science, 287(5456), 1269-1272. [PubMed: 10678834]

Huang W, Wu X, Hu L, Wang L, Ding Y, \& Qu Z (2017). Revisiting the earliest electrophysiological correlate of familiar face recognition. Int J Psychophysiol, 120, 42-53. doi:10.1016/j.ijpsycho. 2017.07.001 [PubMed: 28684327]

Kadipasaoglu CM, Conner CR, Baboyan VG, Rollo M, Pieters TA, \& Tandon N (2017). Network dynamics of human face perception. PLoS One, 12(11), e0188834. doi:10.1371/journal.pone. 0188834 [PubMed: 29190811]

Kaufmann JM, Schweinberger SR, \& Burton MA (2009). N250 ERP correlates of the acquisition of face representations across different images. Journal of Cognitive Neuroscience, 21(4), 625-641. [PubMed: 18702593]

Kaufmann JM, \& Schweinberger SR (2012). The faces you remember: Caricaturing shape facilitates brain processes reflecting the acquisition of new face representations. Biological psychology, 89(1), 21-33. [PubMed: 21925235]

Kramer RSS, Manesi Z, Towler A, Reynolds MG, \& Burton AM (2017). Familiarity and WithinPerson Facial Variability: The Importance of the Internal and External Features. Perception, 301006617725242. doi:10.1177/0301006617725242

Landi SM, \& Freiwald WA (2017). Two areas for familiar face recognition in the primate brain. Science, 357(6351), 591-595. doi:10.1126/science.aan1139 [PubMed: 28798130]

Leveroni CL, Seidenberg M, Mayer AR, Mead LA, Binder JR, \& Rao SM (2000). Neural systems underlying the recognition of familiar and newly learned faces. Journal of Neuroscience, 20, 878886. [PubMed: 10632617]

Lithfous S, \& Rossion B (2018). Electrophysiological individual face adaptation effects with fast periodic visual stimulation resist long interruptions in adaptation. Biological psychology, 133, 4-9. [PubMed: 29373822]

Maris E, \& Oostenveld R (2007). Nonparametric statistical testing of EEG-and MEG-data. Journal of neuroscience methods, 164(1), 177-190. [PubMed: 17517438]

Marti S, \& Dehaene S (2017). Discrete and continuous mechanisms of temporal selection in rapid visual streams. Nature communications, 8(1), 1955.

Megreya AM, \& Burton AM (2006). Unfamiliar faces are not faces: Evidence from a matching task. Memory \& Cognition, 34, 865-876. [PubMed: 17063917]

Müller MM, Andersen S, Trujillo NJ, Valdes-Sosa P, Malinowski P, \& Hillyard SA (2006). Featureselective attention enhances color signals in early visual areas of the human brain. Proceedings of the National Academy of Sciences, 103(38), 14250-14254.

Nakamura K, Kawashima R, Sato N, Nakamura A, Sugiura M, Kato T, .. \& Zilles K (2000). Functional delineation of the human occipito-temporal areas related to face and scene processing: a PET study. Brain, 123(9), 1903-1912. [PubMed: 10960054] 
Nemrodov D, Jacques C, \& Rossion B (2015). Temporal dynamics of repetition suppression to individual faces presented at a fast periodic rate. International Journal of Psychophysiology, 98(1), 35-43. [PubMed: 26113059]

Norcia AM, Appelbaum LG, Ales JM, Cottereau BR, \& Rossion B (2015). The steady-state visual evoked potential in vision research: a review. Journal of vision, 15(6), 4-4.

Oostenveld R, \& Praamstra P (2001). The five percent electrode system for high-resolution EEG and ERP measurements. Clinical neurophysiology, 112(4), 713-719. [PubMed: 11275545]

O'toole AJ, Deffenbacher KA, Valentin D, \& Abdi H (1994). Structural aspects of face recognition and the other-race effect. Memory \& Cognition, 22(2), 208-224. [PubMed: 8035697]

Pourtois G, Schwartz S, Seghier ML, Lazeyras FO, \& Vuilleumier P (2005). View-independent coding of face identity in frontal and temporal cortices is modulated by familiarity: An event-related fMI study. Neuroimage, 24, 1214-1224. [PubMed: 15670699]

Quek GL, \& Rossion B (2017). Category-selective human brain processes elicited in fast periodic visual stimulation streams are immune to temporal predictability. Neuropsychologia, 104, 182200. [PubMed: 28811258]

Quek G, Nemdorov D, Rossion B, \& Liu-Shuang J (2018). Selective attention to faces in a rapid visual stream: Hemispheric differences in enhancement and suppression of category-selective neural activity. Journal of cognitive neuroscience, 30(3), 393-410. [PubMed: 29257716]

Ramon M, Vizioli L, Liu-Shuang J, \& Rossion B (2015). Neural microgenesis of personally familiar face recognition. Proc Natl Acad Sci USA. doi:10.1073/pnas.1414929112

Regan D (1966). Some characteristics of average steady-state and transient responses evoked by modulated light. Electroencephalography and clinical neurophysiology, 20(3), 238-248. [PubMed: 4160391]

Retter TL, \& Rossion B (2017). Visual adaptation reveals an objective electrophysiological measure of high-level individual face discrimination. Scientific Reports, 7.

Robinson AK, Praveen V, Boring M, Tarr M, Grover P, Behrmann M (2017) Very high density EEG elucidates spatiotemporal aspects of early visual processing. Scientific Reports. 7, 16248. [PubMed: 29176609]

Rossion B (2014). Understanding individual face discrimination by means of fast periodic visual stimulation. Experimental Brain Research, 232(6), 1599-1621. [PubMed: 24728131]

Rossion B, \& Boremanse A (2011). Robust sensitivity to facial identity in the right human occipitotemporal cortex as revealed by steady-state visual-evoked potentials. Journal of Vision, 11(2), 1616.

Rossion B, Schiltz C, Robaye L, Pirenne D, \& Crommelinck M (2001). How does the brain discriminate familiar and unfamiliar faces?: A PET study of categorical perception. Journal of Cognitive Neuroscience, 13, 1019-1034. [PubMed: 11595103]

Rotshtein P, Henson RN, Treves A, Driver J, \& Dolan RJ (2005). Morphing Marilyn into Maggie dissociates physical and identity face representations in the brain. Nature neuroscience, 8(1), 107113. [PubMed: 15592463]

Sadeh B, Podlipsky I, Zhdanov A, \& Yovel G (2010). Event-related potential and functional MRI measures of face-selectivity are highly correlated: a simultaneous ERP-fMRI investigation. Hum Brain Mapp, 31(10), 1490-1501. doi:10.1002/hbm.20952 [PubMed: 20127870]

Sergent J, Ohta S, \& MacDonald B (1992). Functional neuroanatomy of face and object processing: a positron emission tomography study. Brain, 115(1), 15-36. [PubMed: 1559150]

Shapiro KL, Raymond JE, \& Arnell KM (1994). Attention to visual pattern information produces the attentional blink in rapid serial visual presentation. Journal of Experimental psychology: Human perception and performance, 20(2), 357. [PubMed: 8189198]

Smith ML, Volna B, \& Ewing L (2016). Distinct information critically distinguishes judgments of face familiarity and identity. J Exp Psychol Hum Percept Perform, 42(11), 1770-1779. doi:10.1037/ xhp0000243 [PubMed: 27379874]

Schweinberger SR, Pickering EC, Jentzsch I, Burton AM, \& Kaufmann JM (2002). Event-related brain potential evidence for a response of inferior temporal cortex to familiar face repetitions. Cognitive Brain Research, 14(3), 398-409. [PubMed: 12421663] 
Tanaka JW, Curran T, Porterfield AL, \& Collins D (2006). Activation of preexisting and acquired face representations: the N250 event-related potential as an index of face familiarity. Journal of Cognitive Neuroscience, 18(9), 1488-1497. [PubMed: 16989550]

Visconti di Oleggio Castello M, \& Gobbini MI (2015). Familiar Face Detection in 180ms. PLoS One, 10(8), e0136548. doi:10.1371/journal.pone.0136548 [PubMed: 26305788]

Weibert K, \& Andrews TJ (2015). Activity in the right fusiform face area predicts the behavioural advantage for the perception of familiar faces. Neuropsychologia, 75, 588-596. doi:10.1016/ j.neuropsychologia.2015.07.015 [PubMed: 26187507]

Weibert K, Harris RJ, Mitchell A, Byrne H, Young AW, \& Andrews TJ (2016). An image-invariant neural response to familiar faces in the human medial temporal lobe.Cortex, 84, 34-42. doi: 10.1016/j.cortex.2016.08.014 [PubMed: 27697662]

Wieser MJ, \& Keil A (2014). Fearful faces heighten the cortical representation of contextual threat. NeuroImage, 86, 317-325. [PubMed: 24125792]

Wieser MJ, Miskovic V, Rausch S, \& Keil A (2014). Different time course of visuocortical signal changes to fear-conditioned faces with direct or averted gaze: A ssVEP study with single-trial analysis. Neuropsychologia, 62, 101-110. [PubMed: 25050854]

Xu B, Liu-Shuang J, Rossion B, Tanaka J (2017). Individual differences in face identity processing with face periodic visual stimulation. Journal of Cognitive Neuroscience, 29:8, 1368-1377

Young AW, \& Burton AM (2017). Are We Face Experts?. Trends in cognitive sciences.

Zimmermann FG, \& Eimer M (2013). Face learning and the emergence of view-independent face recognition: an event-related brain potential study. Neuropsychologia, 51(7), 1320-1329.

[PubMed: 23583970]

Neuroimage. Author manuscript; available in PMC 2019 November 01. 


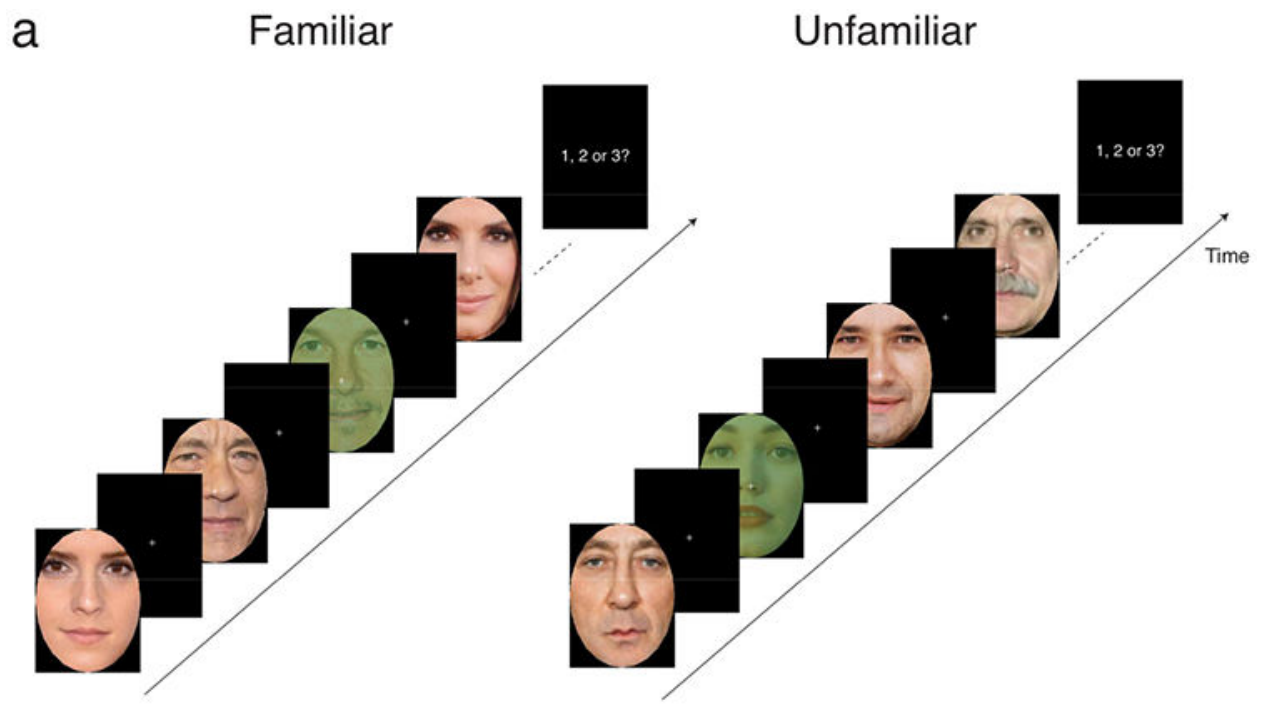

b
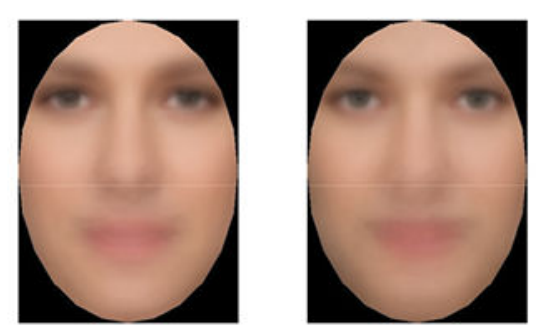

C
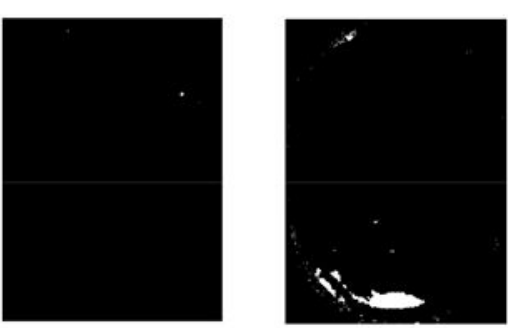

Figure 1.

a) Experimental design. Participants viewed 60 second blocks consisting of sequentially presented familiar or unfamiliar faces. Faces were presented at either $15 \mathrm{~Hz}, 6 \mathrm{~Hz}$ or $3.75 \mathrm{~Hz}$ in upright or inverted orientation. Participants had to count the number of faces that appeared with a green tint (1,2 or 3 during the block). b) Mean images created from all familiar faces (left) and all unfamiliar faces (right). c) Pixels with significantly higher luminance for unfamiliar > familiar images (left) and familiar > unfamiliar images (right), $p$ $<.001$, uncorrected. 
a
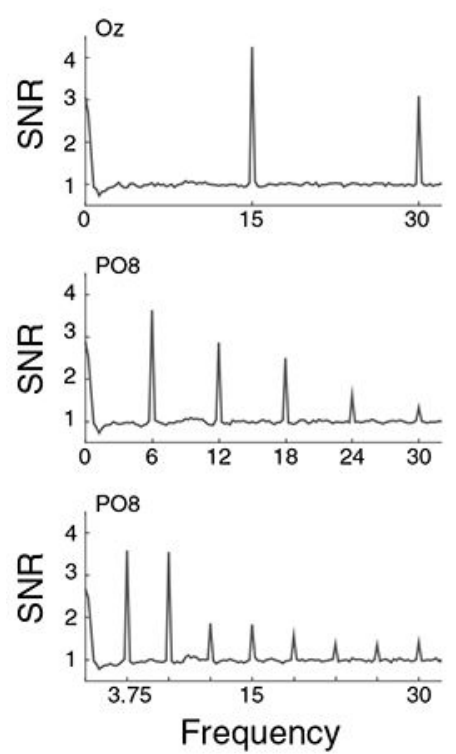

b

Upright

Inverted

$15 \mathrm{~Hz}$

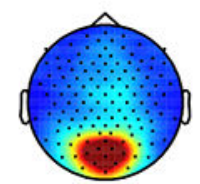

$6 \mathrm{~Hz}$
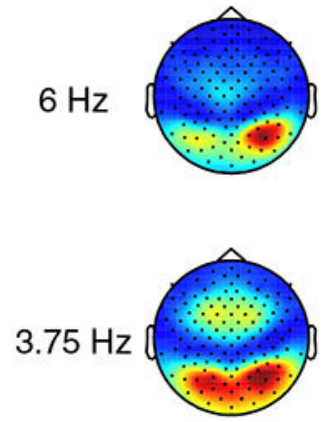

Unfamiliar
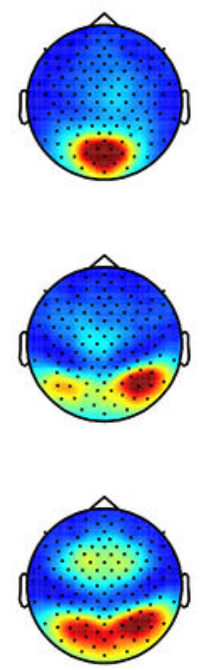

Familiar
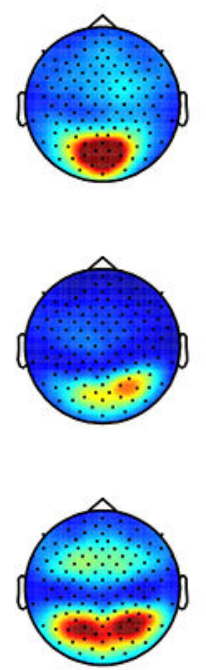

Unfamiliar
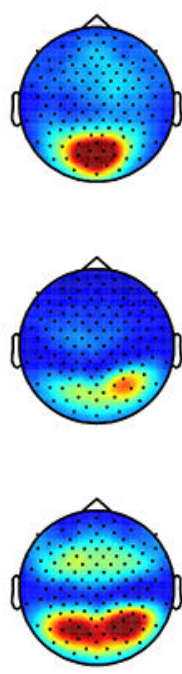

Figure 2.

Signal to noise ratio (SNR) of periodic signal. a) Mean SNR spectra for upright unfamiliar faces presented at $15 \mathrm{~Hz}, 6 \mathrm{~Hz}$ and $3.75 \mathrm{~Hz}$ frequencies. Peaks are evident at the presentation frequencies and their harmonics. b) Head maps showing mean signal for the three flicker frequency conditions $(15 \mathrm{~Hz}, 6 \mathrm{~Hz}, 3.75 \mathrm{~Hz})$. From left to right, head maps correspond with responses to upright familiar faces, upright unfamiliar faces, inverted familiar faces, and inverted unfamiliar faces at the fundamental frequency. Noise level SNR is 1. Black dots indicate clusters of electrodes with significant signal SNR (above 1). All conditions showed significant signal at the image presentation frequency, $p \mathrm{~s}<.001$. 


\section{Familiar - Unfamiliar}

Upright

Inverted

$15 \mathrm{~Hz}$
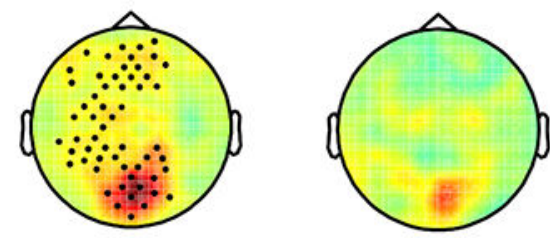

$6 \mathrm{~Hz}$
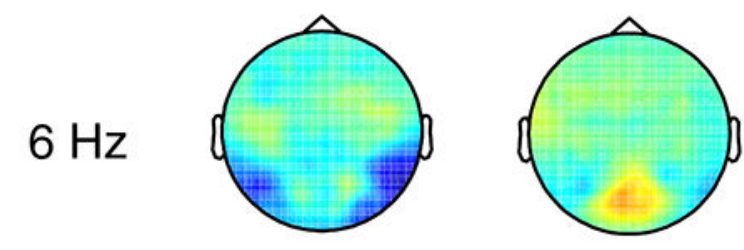

0.5
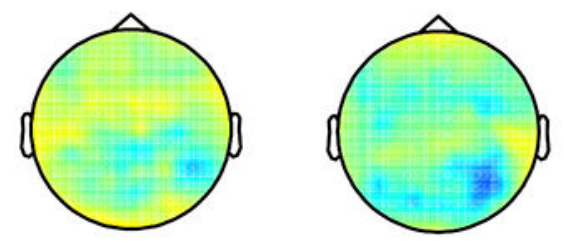

Figure 3.

Differences in SNR between familiar and unfamiliar faces. Black dots indicate clusters of electrodes with significant differences across conditions. At the $15 \mathrm{~Hz}$ frequency, SNR was larger for upright familiar faces than for upright unfamiliar faces. At the $6 \mathrm{~Hz}$ frequency, SNR was marginally lower for familiar faces. Finally, the $3.75 \mathrm{~Hz}$ frequency exhibited no effects of familiarity. There were no differences in SNR for inverted faces at any frequency. 
a

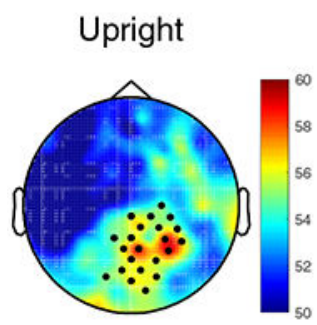

C

Familiar

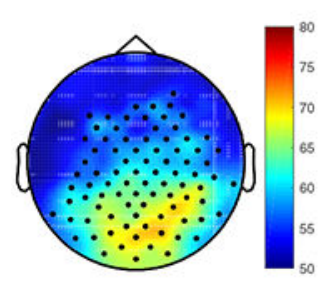

Familiarity Decoding

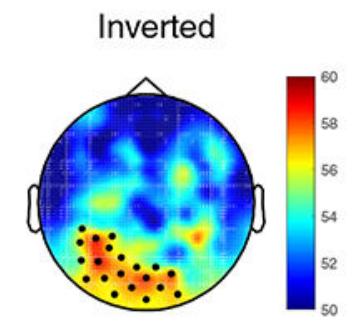

Orientation Decoding

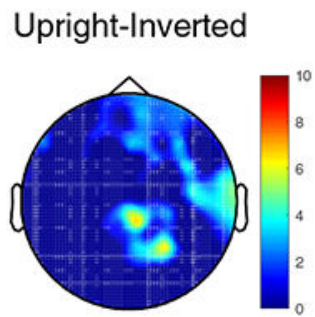

b

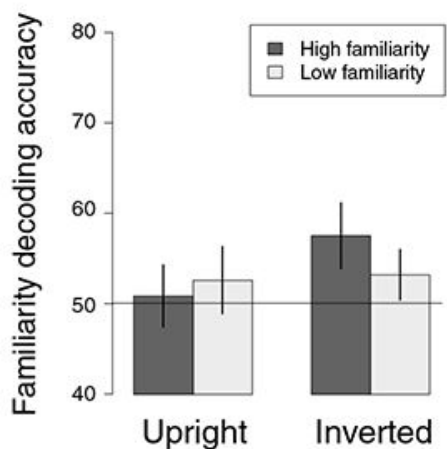

d

Familiar-Unfamiliar
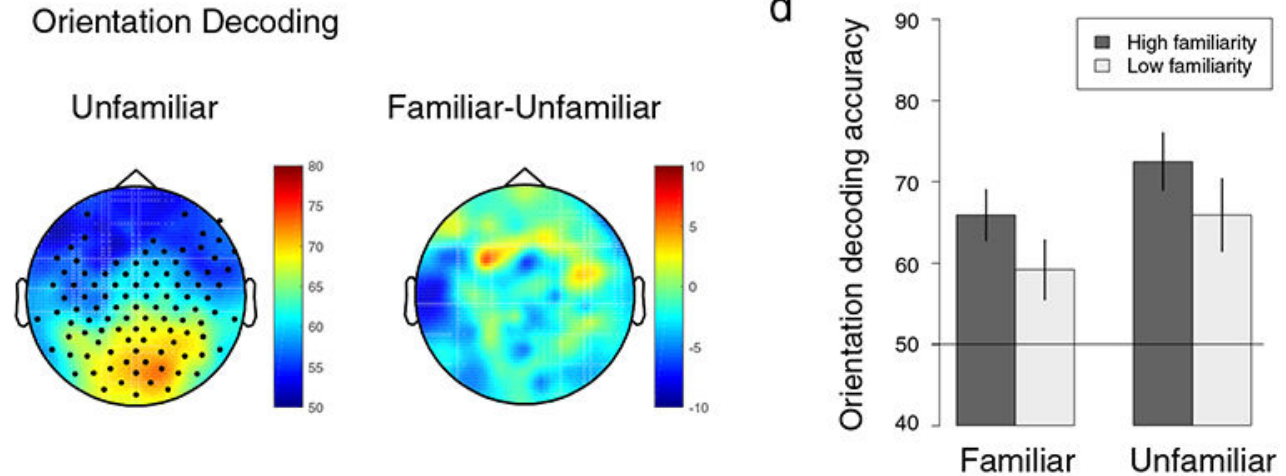

Figure 4.

Multivariate decoding results for $15 \mathrm{~Hz}$ frequency. a) Decoding familiar versus unfamiliar faces using searchlight method. b) Whole brain familiarity decoding results as a function of face orientation and participant familiarity ratings. c) Decoding upright versus inverted faces using searchlight method. d) Whole brain orientation decoding results as a function of face familiarity and participant familiarity ratings. 
a

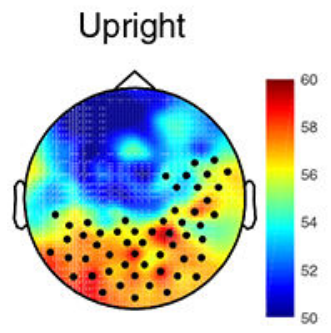

C
Familiarity Decoding

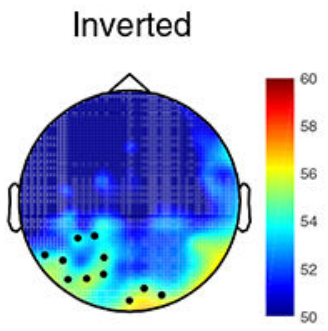

Orientation Decoding

Unfamiliar

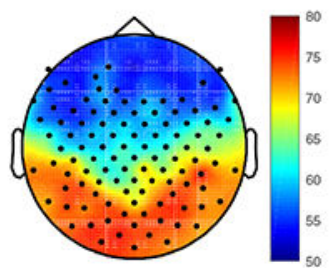

b
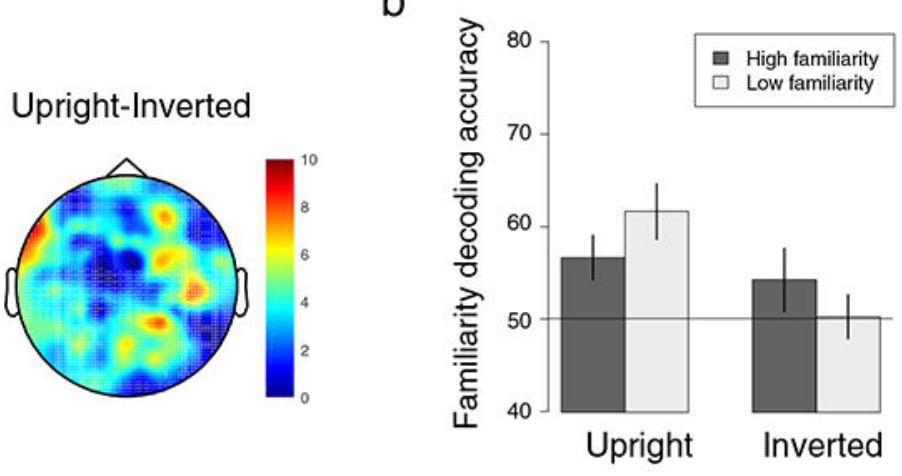

d

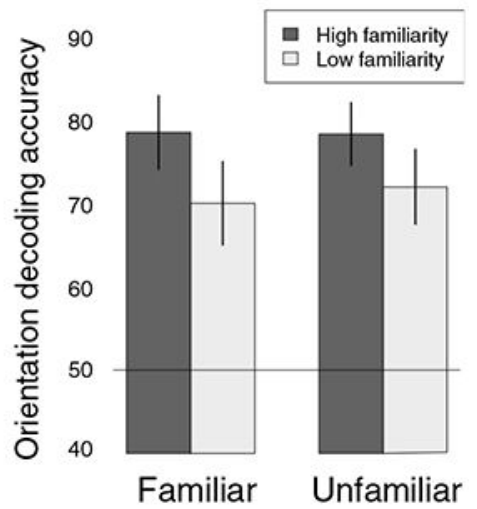

Figure 5.

Multivariate decoding results for $6 \mathrm{~Hz}$ frequency. a) Decoding familiar versus unfamiliar faces using searchlight method. Decoding accuracy was significantly above chance for both upright and inverted faces. b) Whole brain familiarity decoding results as a function of face orientation and participant familiarity ratings. c) Decoding upright versus inverted faces using searchlight method. Orientation decoding was better over posterior than frontal clusters. Black dots signify significant clusters of electrodes with above chance decoding. d) Whole brain orientation decoding results as a function of face familiarity and participant familiarity ratings. 
a

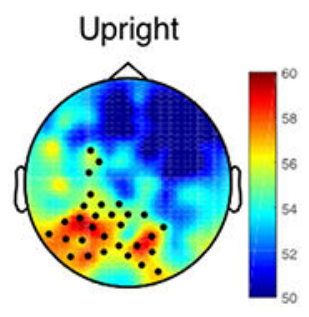

C

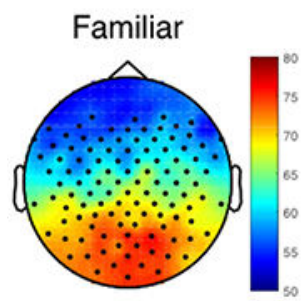

Familiarity Decoding

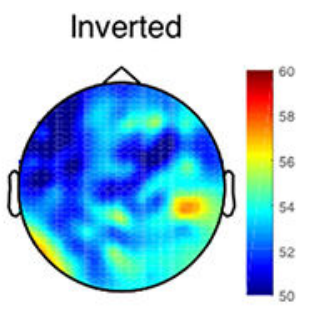

Orientation Decoding

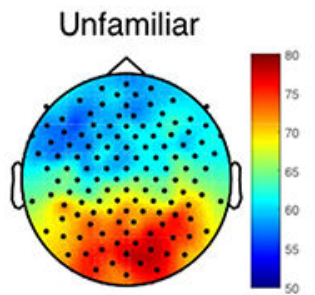

b
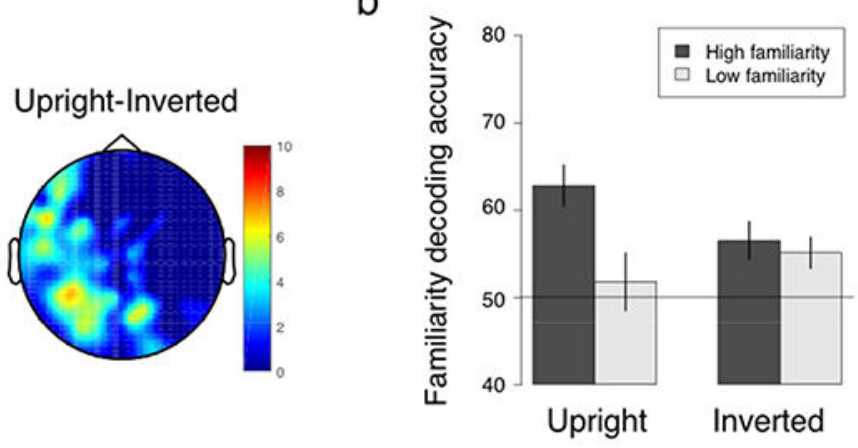

d

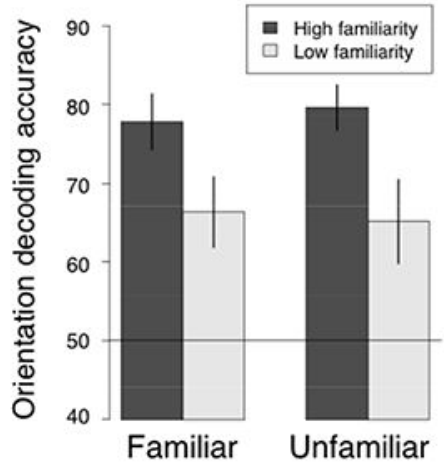

Figure 6.

Results for $3.75 \mathrm{~Hz}$ frequency. a) Decoding familiar versus unfamiliar faces using searchlight method. Significant decoding was observed for upright, but not inverted, faces. b) Whole brain familiarity decoding results as a function of face orientation and participant familiarity ratings. Participants with high familiarity had significantly better decoding for upright faces. c) Decoding upright versus inverted faces using searchlight method. Black dots signify significant clusters of electrodes with above chance decoding. d) Whole brain orientation decoding results as a function of face familiarity and participant familiarity ratings. Participants with high familiarity had better orientation decoding. 
Table 1.

Summary table for the difference between familiar and unfamiliar faces in terms of signal strength, decoding accuracy and familiarity ratings. "Univariate difference" $p$-values are cluster-based statistics for the SNR difference between familiar and unfamiliar faces at the respective presentation frequencies. "Searchlight decoding" values are cluster-based statistics for decoding accuracy of familiar versus unfamiliar faces compared with chance (50\%). "Decoding: High vs low familiarity" denotes difference between participants with low familiarity ratings and those with high familiarity ratings for whole brain familiarity decoding. Effects of familiarity were evident at all frequencies, but the patterns varied due to differences in the level of processing at each frequency. Familiarity effects were much more evident for upright faces than inverted faces.

\begin{tabular}{lllclcc}
\hline & \multicolumn{5}{c}{ Gamiliar versus unfamiliar faces } \\
\cline { 2 - 6 } & Univariate difference & Searchlight decoding & $\begin{array}{c}\text { Decoding: High } \\
\text { vs low familiarity } \\
\text { ratings }\end{array}$ & Univariate & Searchlight decoding & $\begin{array}{c}\text { Decoding: High } \\
\text { vs low familiarity } \\
\text { ratings }\end{array}$ \\
\hline $15 \mathrm{~Hz}$ & $p=.002$ & $p=.010$ & $p=.732$ & $p>.05$ & $p=.018$ & $p=.361$ \\
\hline $6 \mathrm{~Hz}$ & $p=.055$ & $p<.001$ & $p=.212$ & $p>.05$ & $p=.023$ & $p=.350$ \\
\hline $3.75 \mathrm{~Hz}$ & $p>.05$ & $p=.007$ & $p=.016$ & $p>.05$ & $p>.05$ & $p=.628$ \\
\hline
\end{tabular}

